\title{
Effect of Sumatriptan on Learning and Memory Impairment Resulting from Repetitive Spreading Depression
}

\author{
Mahmoud Lotfinia ${ }^{1,2 *}$, Ahmad Ali Lotfinia ${ }^{1}$, Sina Asaadi ${ }^{2}$ \\ ${ }^{1}$ Shefa Neuroscience Research Center, Khatam Alanbia Hospital, Tehran, Iran. \\ ${ }^{2}$ School of Medicine, Shahid Beheshti University of Medical Sciences, Tehran, Iran.
}

\section{ABSTRACT}

Introduction: Spreading depression (SD) is a transient and self-propagating wave of neuronal and glial depolarization, followed by temporary loss of all brain activities. SD has been implicated in migraine and other headaches and can be evoked in experimental animals by electrical or chemical stimulation. It has been shown that repetitive SD produced memory deficits in juvenile rats. The effect of migraine prophylactic drugs on SD has been reported. In the present study, the effect of sumatriptan, a migraine prophylactic drug, on SD-induced memory impairments in juvenile rats was investigated. Materials and Methods: Wistar rats (60-80 gr) were divided into SD, Sham, 0.1 sumatriptan- and 0.5 sumatriptan-treated groups and SD was induced weekly for four weeks by $\mathrm{KCl}$ (2 M) application. Sumatriptan $(0.1$ and $0.5 \mathrm{mg} / \mathrm{kg})$ was also administrated weekly for 4 weeks in treatment groups. Memory retention of passive avoidance learning in rats was evaluated by shuttle box test in different time points. Results: SD significantly increased the initial latency to enter the dark compartment. SD also impaired the retention of the learned tasks. Administration of low dose sumatriptan caused improvement in memory retention 30 minutes after learning, while the high doses could improve the memory 30 minutes, 24 hours and 1 week after learning. Conclusion: This study shows the positive role of sumatriptan in learning and memory impairment induced by repetitive $\mathrm{SD}$.

\section{Key words:}

1. Cortical Spreading Depression

2. Sumatriptan

3. Avoidance Learning

4. Memory

* Corresponding Author: Mahmoud Lotfinia

E-mail:mdla617@yahoo.com 
بررسى اثر داروى سوماترييتان بر يادكيرى و حافظهُ مختل شده به دنبال القاى مهار منتشر شوندهُ مكرر

\author{
محمود لطفى نيا؟، ‘:، احمد على لطفى نيا'، سينا اسعدى' \\ 1. ا. مركز تحقيقات علوم اعصاب شفا، بيمارستان خاتم الانبياء، تهران، ايران.

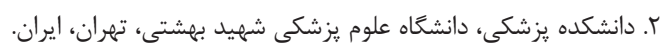

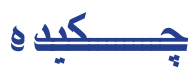

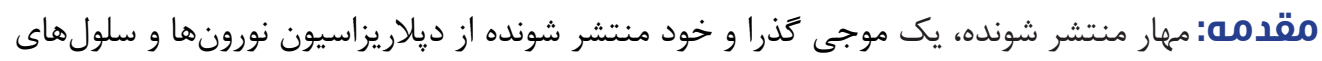

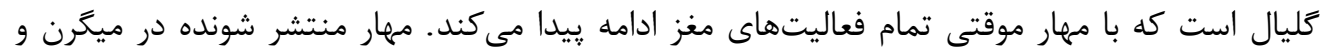

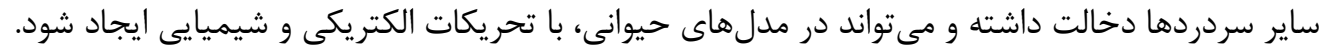

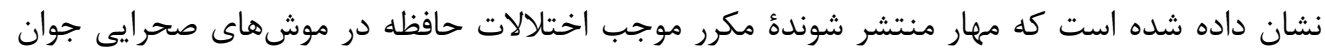

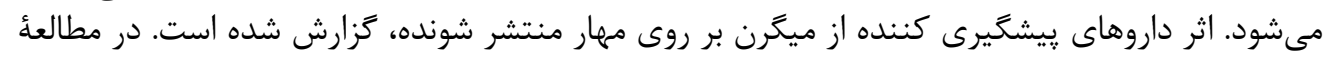

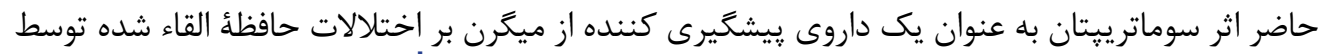

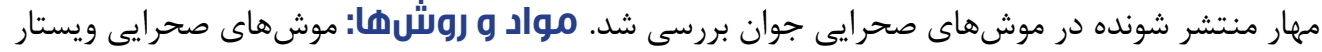

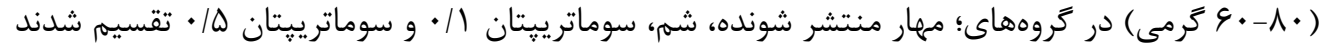

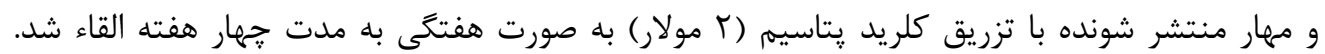

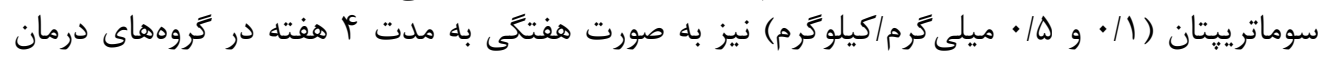

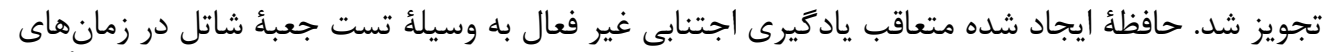

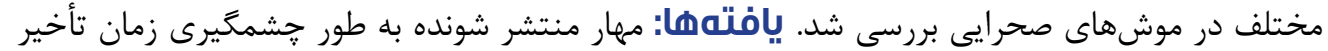

كليد وازهها: 1. مهار منتشر شوندة قشرى

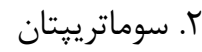

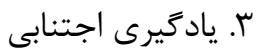
r. r. حافظه

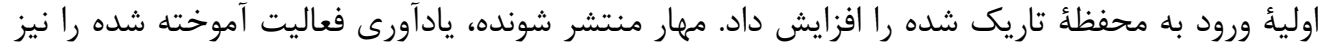

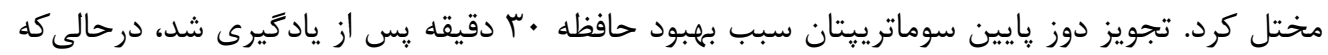

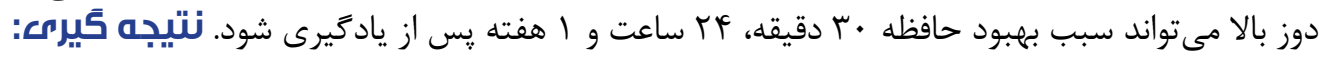

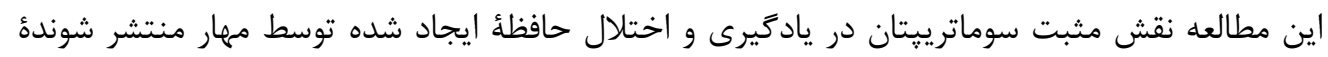
مكرر را نشان داد.

" *ويسنده مسئول: محمود لطفى نيا آدرس الكترونيكى: mdla617@yahoo.com 


\section{مواد و روشها - - ماد \\ حيوانات و گروههاى مورد آزمايش لتر}

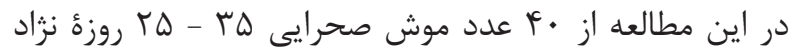

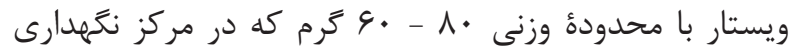

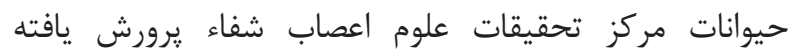

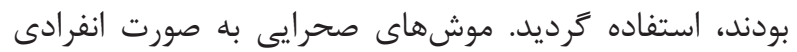

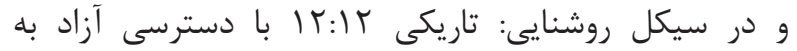

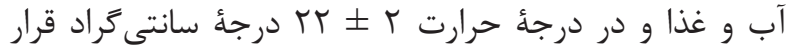

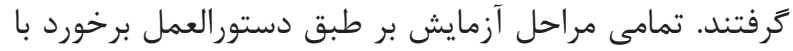

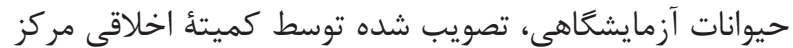

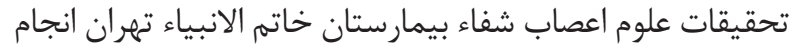

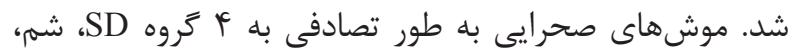

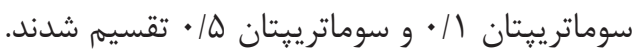
تزريق كلريد يتاسيم و داروى سوماتريتان:

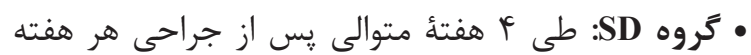

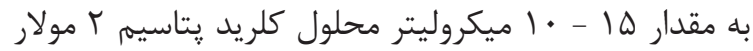

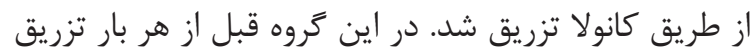

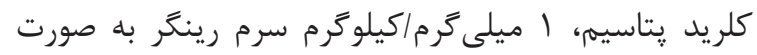
داخل صفاقى تزريق گرديد.

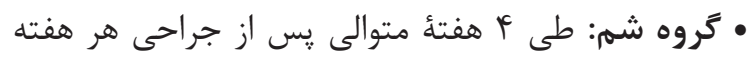

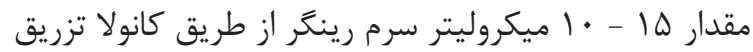
كرديد.

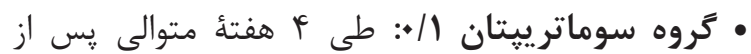

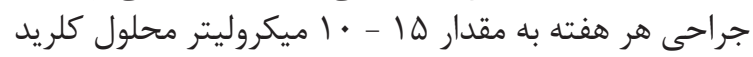

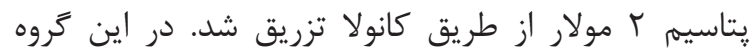

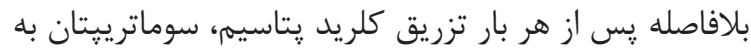

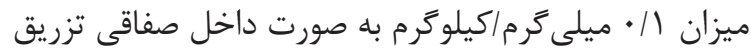
كرديد.

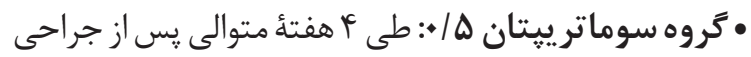

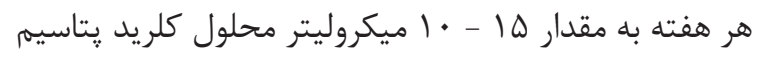

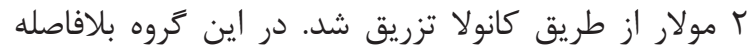

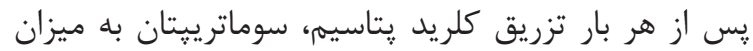

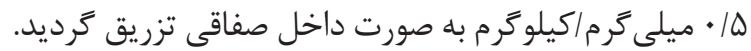

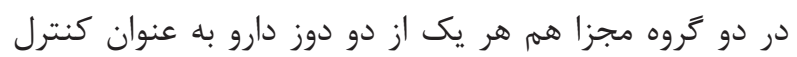

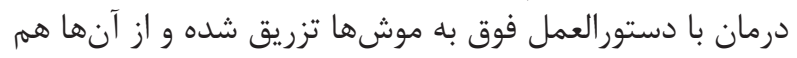

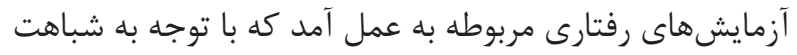

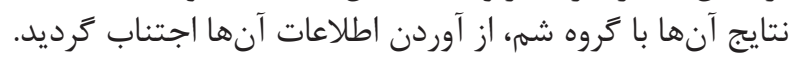

$$
\text { جراحى استريوتاكسى }
$$

در ابتدا موشهاى صحرايى با استفاده از يك ترازوى حسى حساس،

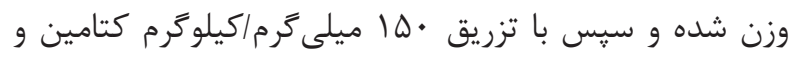

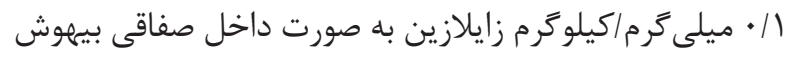

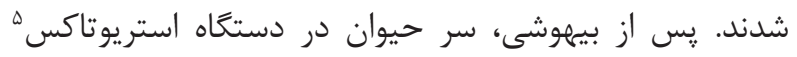

${ }^{1}$ Spreading Depression

${ }^{2}$ Nifedipine

${ }^{3}$ Sumatriptan

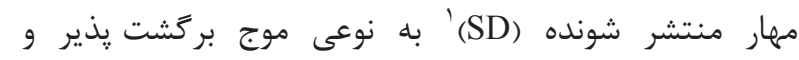

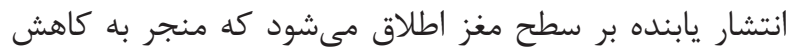

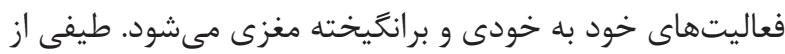

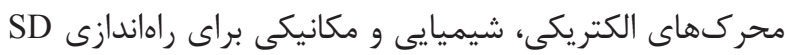

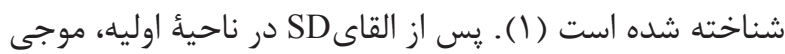

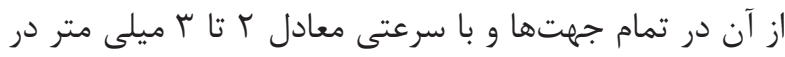

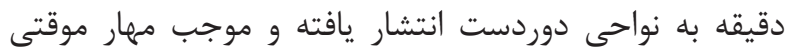

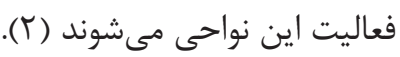

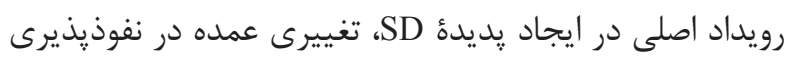

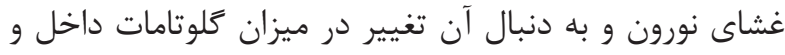

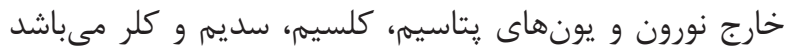

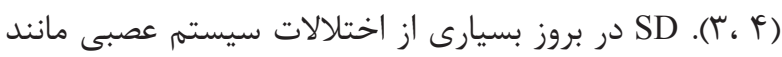

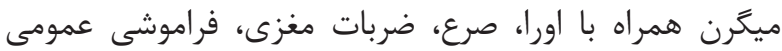

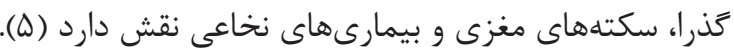
اعتقاد بر آن است كه بر خلاف شرايط فيزيولوزيك، SD قادر

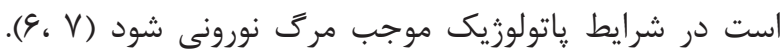

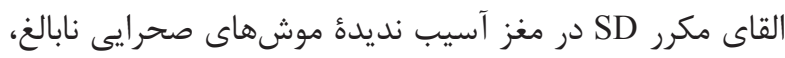

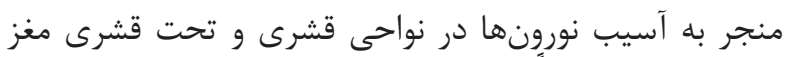

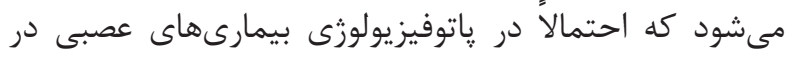

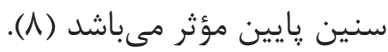

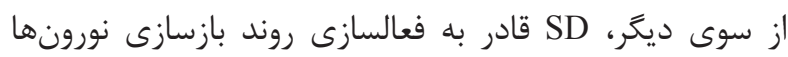

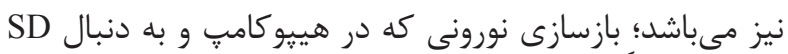

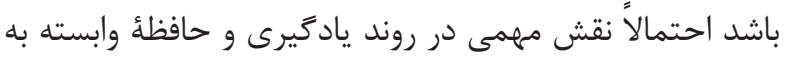

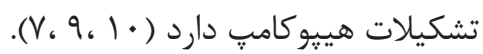

با وجود مطالعات متعددى كه در زمينهُ شناخت SD انجام شده

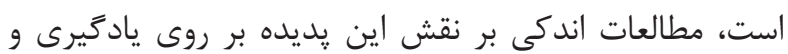

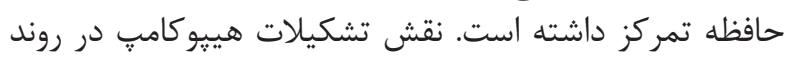

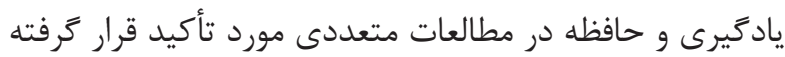

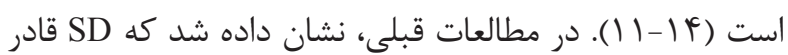

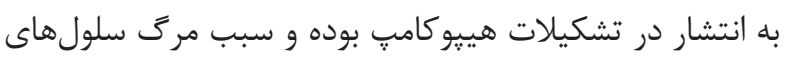

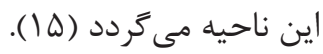

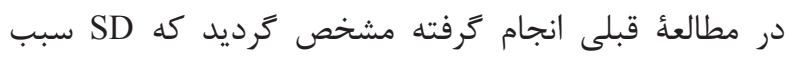

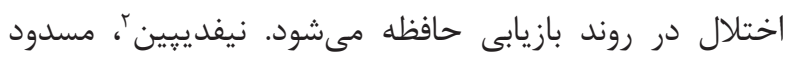

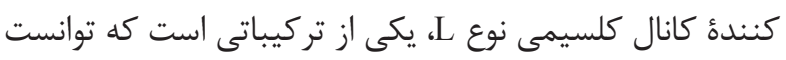

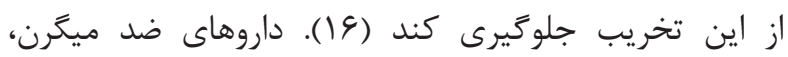

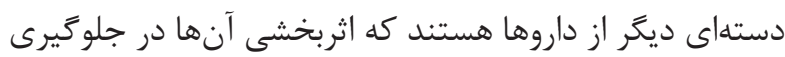

از روند ايجاد و ييشروى SD نشان داده شده است (V) (I).

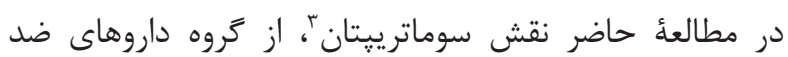

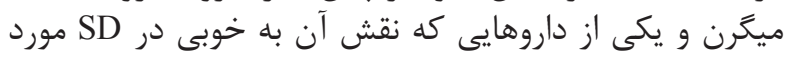

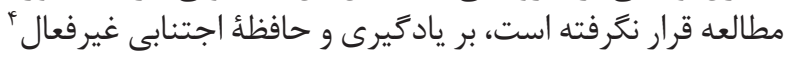
مورد بررسى قرار گرفته است.

\footnotetext{
${ }^{4}$ Passive avoidance learning and memory

${ }^{5}$ Stereotax- Stoelting Instruments, USA
} 


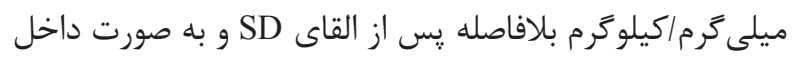

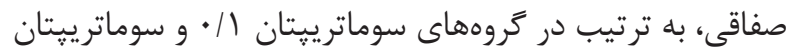

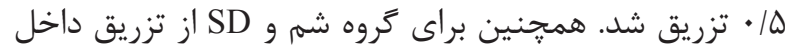

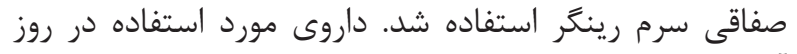

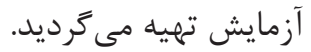

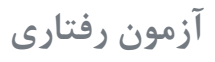

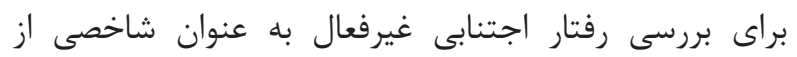

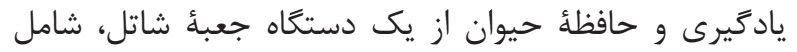

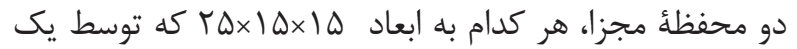

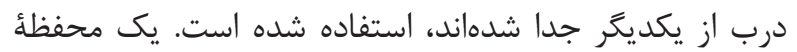

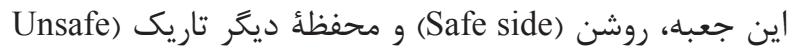

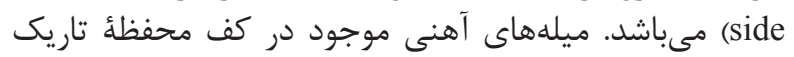

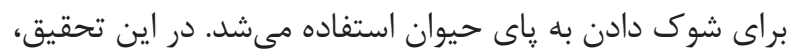

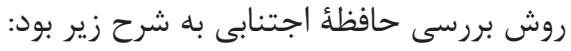

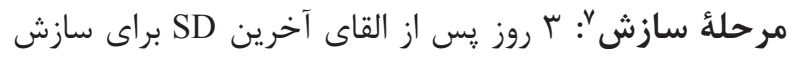

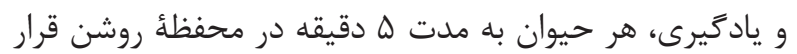

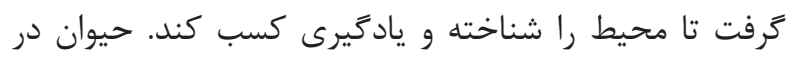

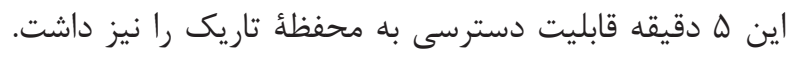

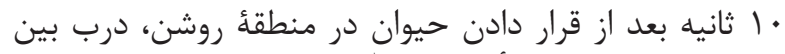

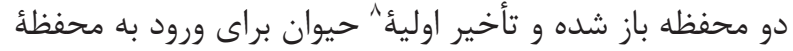

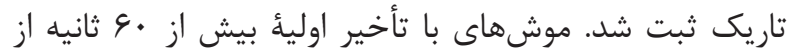

مطالعه خارج شدند.

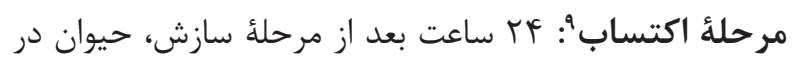

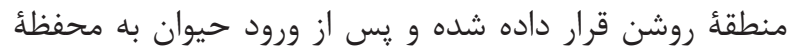

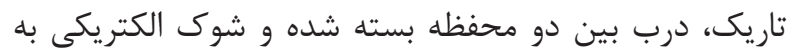

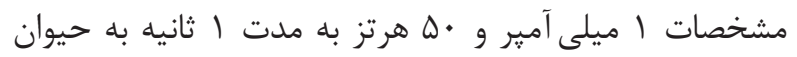

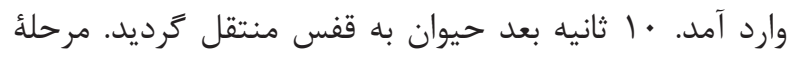

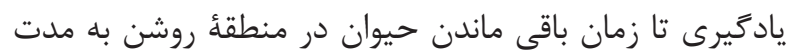

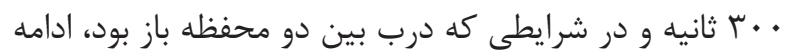

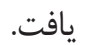

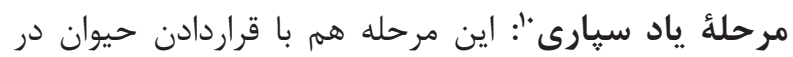

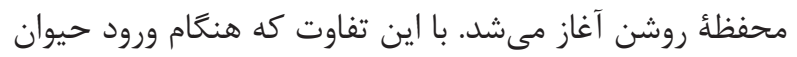

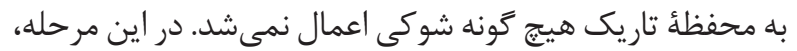

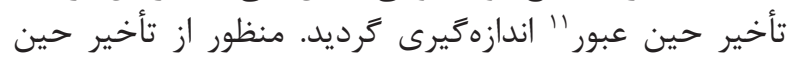

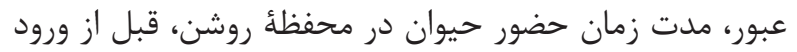

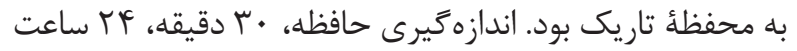

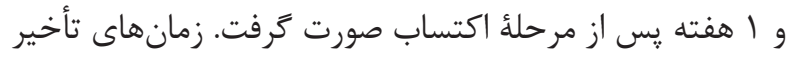

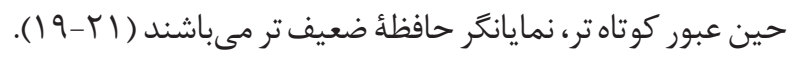

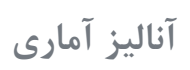

نتايج به دست آمده از آزمون رفتارى توسط نرم افزار IBM SPSS Statistics 20

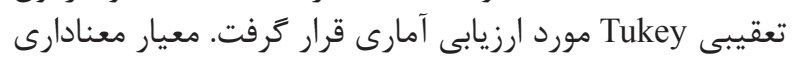

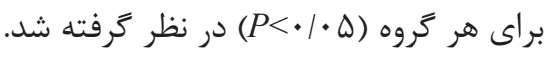

${ }^{6}$ Pentobarbital

${ }^{7}$ Adaptation

${ }^{8}$ Initial latency

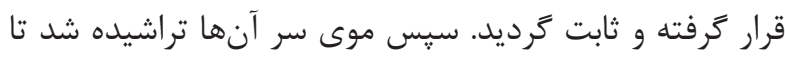

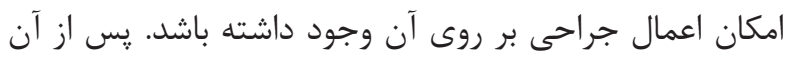

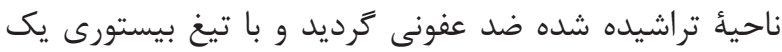

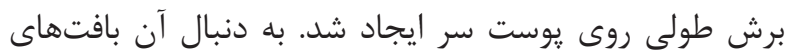

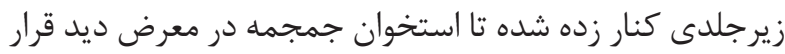

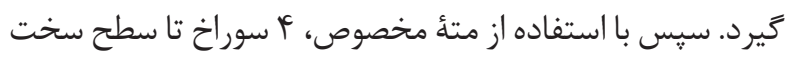

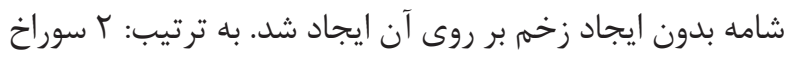

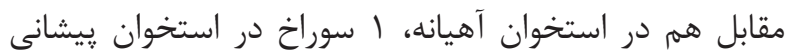

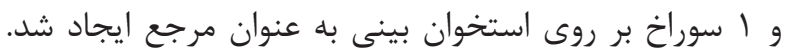

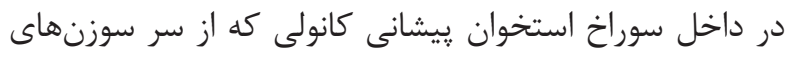

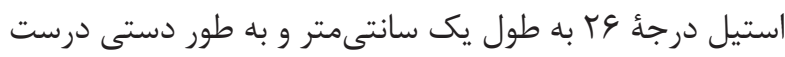

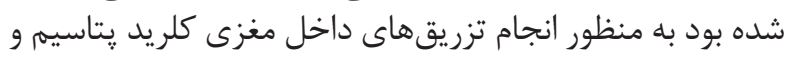

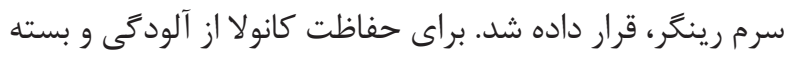

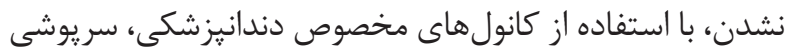

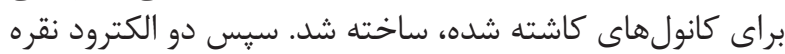

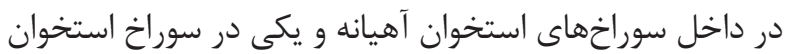

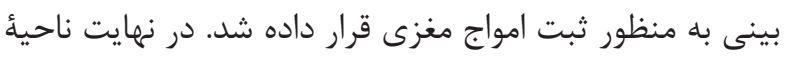
جراحى توسط سيمان جراحى ير شد (1) (1).

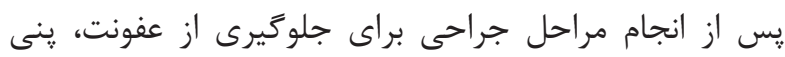

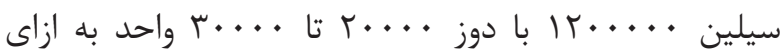
هر كيلوكَرم وزن موش صحرايى استفاده كرديد. يس در از از اتمام

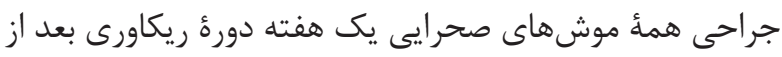

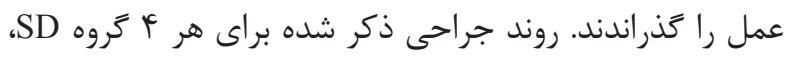

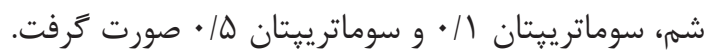

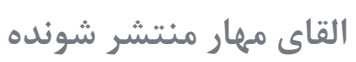

القاى مهار منتشر شونده در سه گروه SD، سوماترييتان / / •

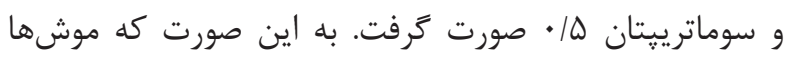

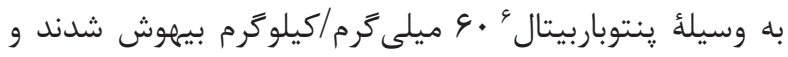

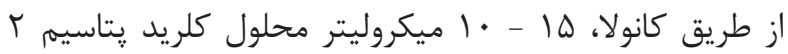

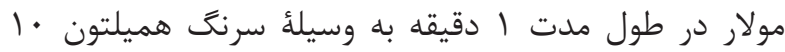

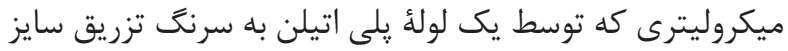

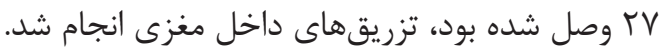

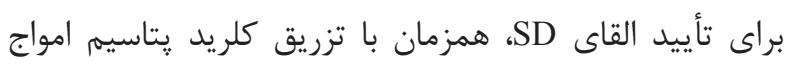

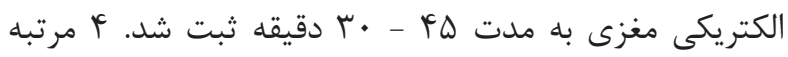

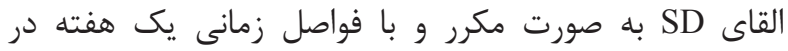

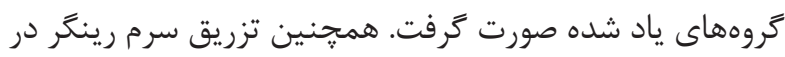
كروه شم از طريق كانولاى كار كذاشته شده، انجام شد فرد

$$
\text { دارو }
$$

داروى سوماترييتان (Sigma)، آكونيست كَيرنده سروتونينى

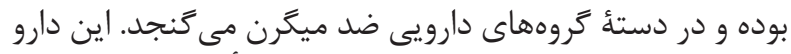

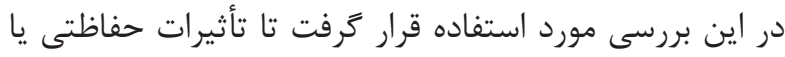

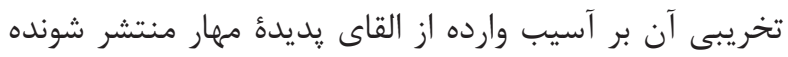

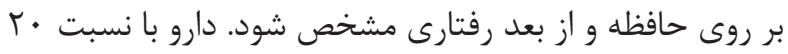

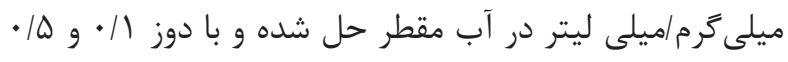

\footnotetext{
${ }^{9}$ Acquisition

${ }^{10}$ Retention

${ }^{11}$ Step-through latency
} 


$$
\text { تأخير حين عبور هي از FF ساعت }
$$

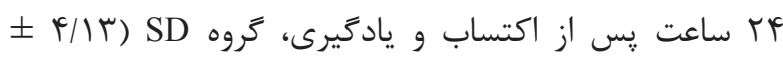
• • داد (YFV/I9

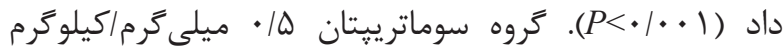

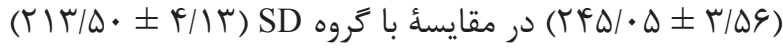

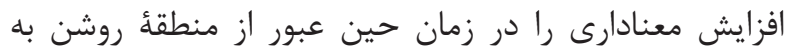

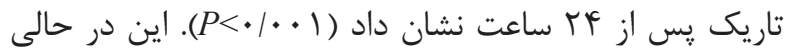

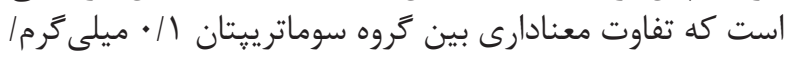
كيلو

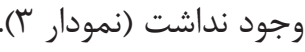

تأخير حين عبور پس از 1 هفته

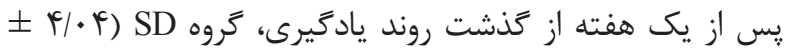

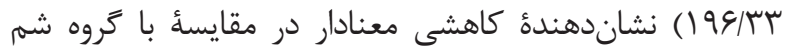

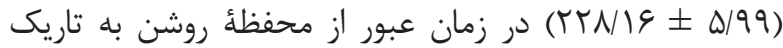

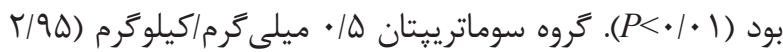

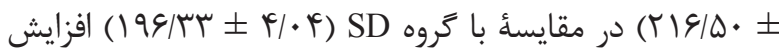

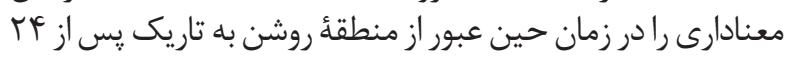

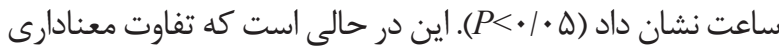

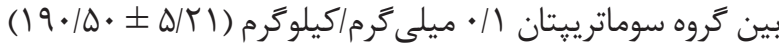

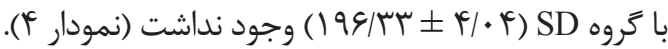

يافتهها

تأخير اوليه

نتايج حاصل از اين بررسى نشان داد كه ميانتين زمان تأخير

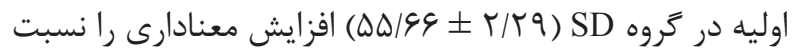

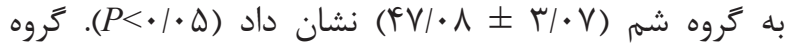

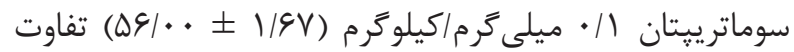

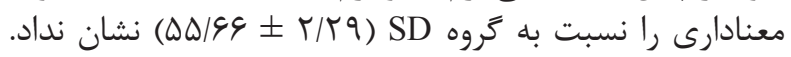

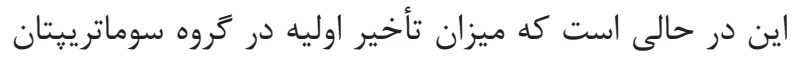

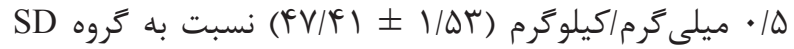

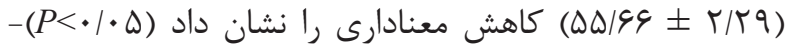

(نمودار (1) (ن) (ن)

تأخير حين عبور يس از • دقيقه

I r

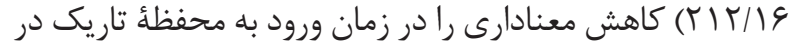

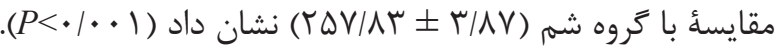

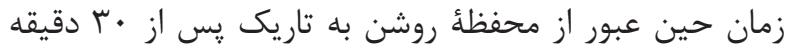

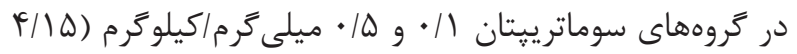

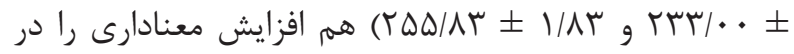

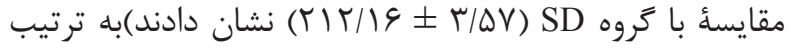
(نمودار (P<) $(P<\cdot 1 \cdot P<\cdot 1 \cdot 1$

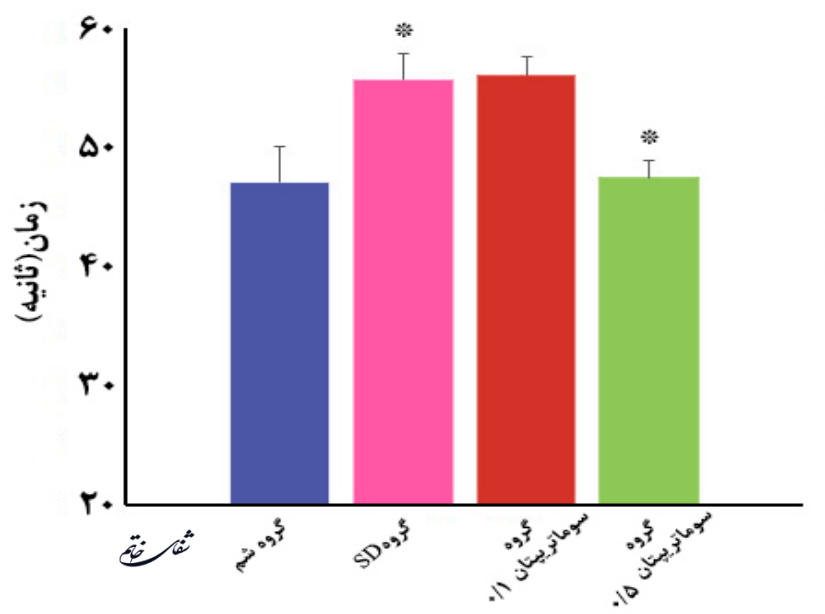

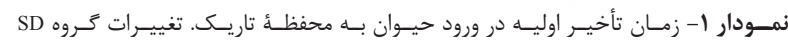

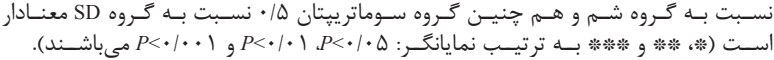

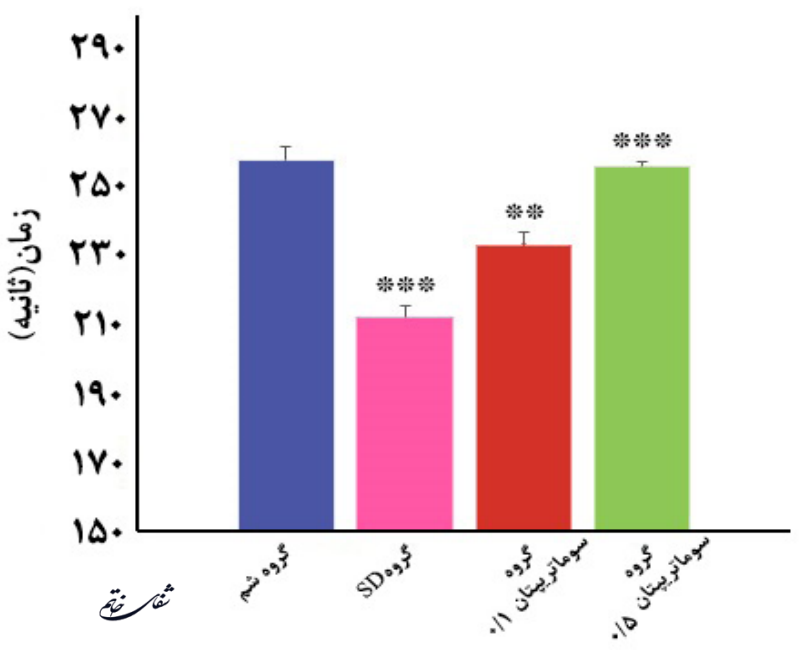



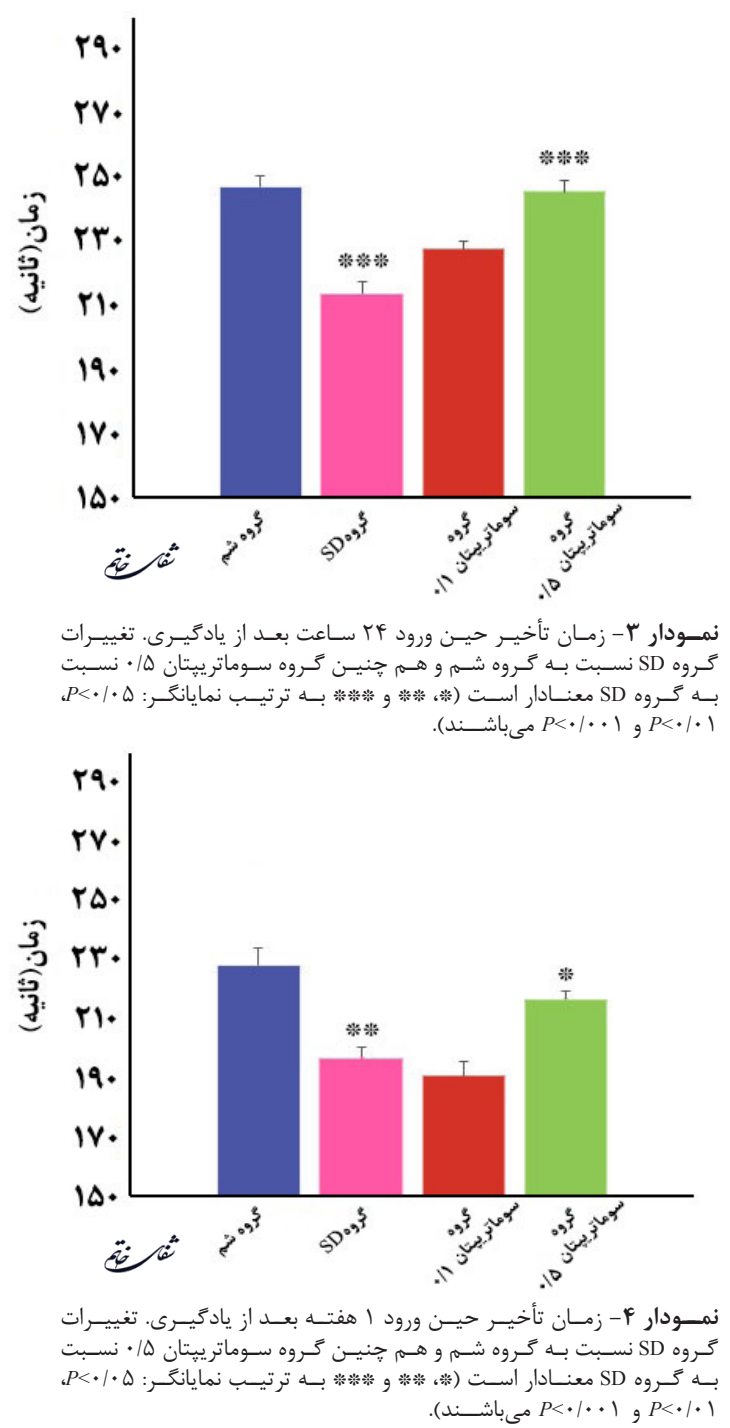

رفته مىباشد. همين موضوع زمينه ساز طراحى و توليد مجموعأ.

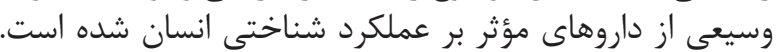

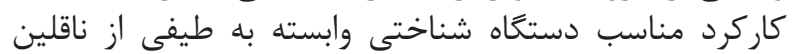

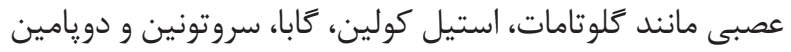

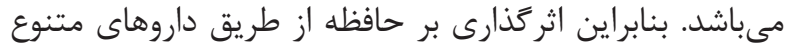

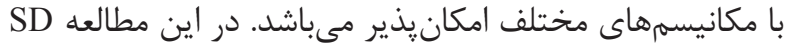

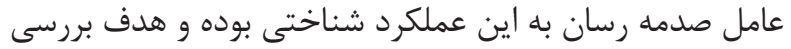
نقش سوماترييتان بر اين روند بودان اين عملينا

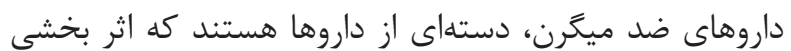

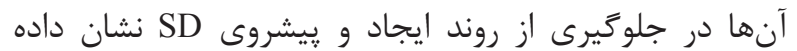

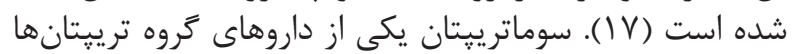

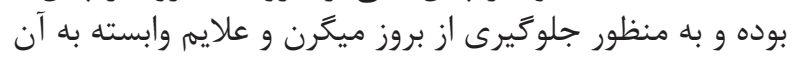

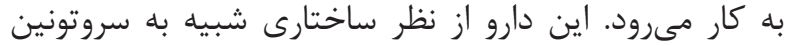

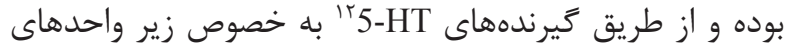
5-HT واحدهاى كيرنده سروتونينى مثل

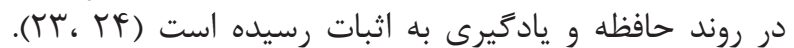

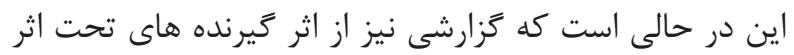

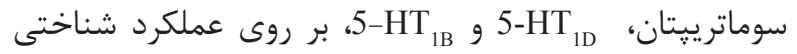
وجود دارد. نشان داده شده است در بيماران دجار ميكرن،
بحث و نتيجه كيرى

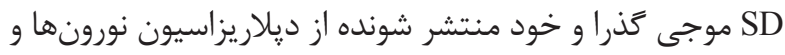

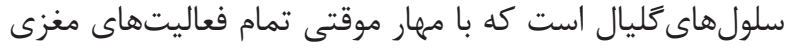

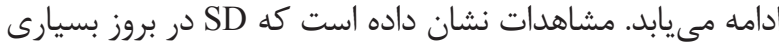

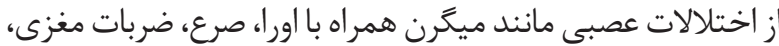

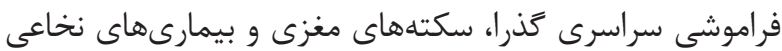

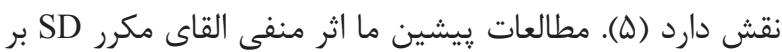

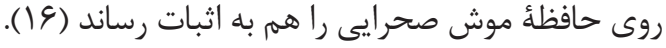

شكل گيرى، يردازش و بازيابى حافظه، وابسته به هييوكامٍ و و

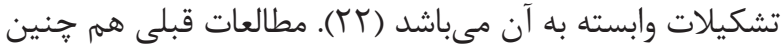

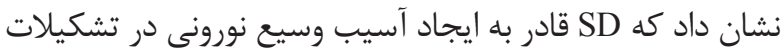

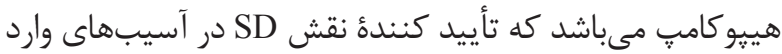

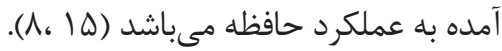

اختلالات شناختى به خصوص اختلال در عملكرد حافظه، از راز

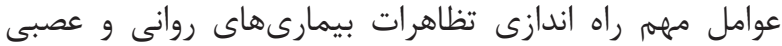

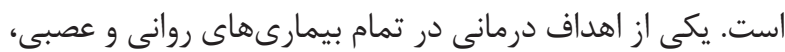

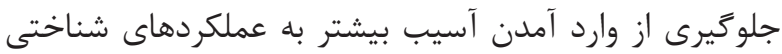

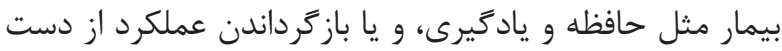


بيان اين گيرندهها در زرء عصبى سه قلو نشان داده شده كه

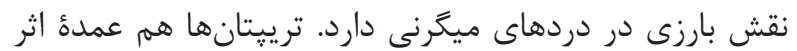

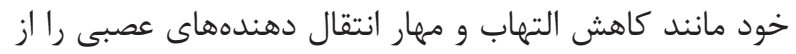

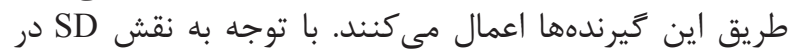

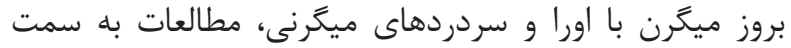
ارتباط بين SD و سروتونين گرايش يافت

مطالعات صورت گرفته نشان داد كه سطوح پايين سروتونين در

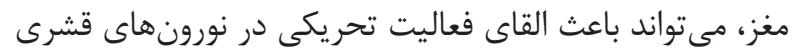

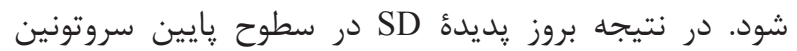

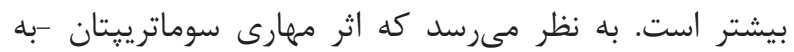

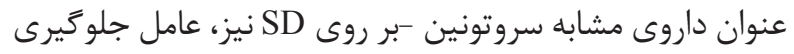

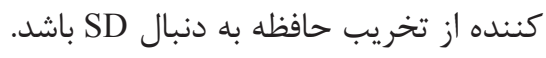

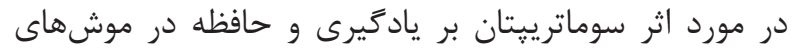

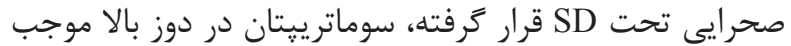

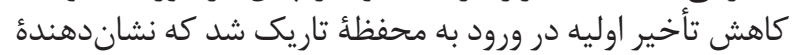

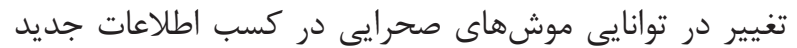

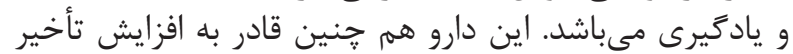

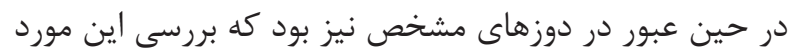

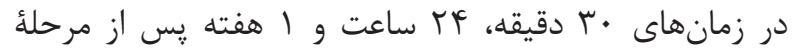

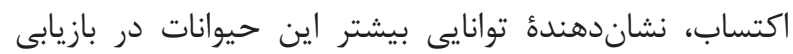

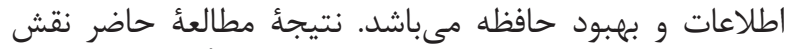

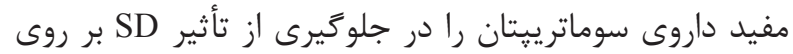

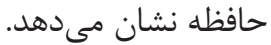

1. Leão AAP. Spreading depression of activity in the cerebral cortex. J Neurophysiol. 1944; 7(6): 359-90.

2. Gorji A. Spreading depression: a review of the clinical relevance. Brain Res Brain Res Rev. 2001;38(1-2):33-60.

3. Kraig RP, Nicholson C. Extracellular ionic variations during spreading depression. Neuroscience. 1978; 3(11): 1045-59.

4. Van Harreveld A. Two mechanisms for spreading depression in the chicken retina. J Neurobiol. 1978; 9(6): 419-31.

5. Lauritzen M, Dreier JP, Fabricius M, Hartings JA, Graf $\mathrm{R}$, Strong AJ. Clinical relevance of cortical spreading depression in neurological disorders: migraine, malignant stroke, subarachnoid and intracranial hemorrhage, and traumatic brain injury. J Cereb Blood Flow Metab. 2011; 31(1): 17-35.

6. Dreier JP, Kleeberg J, Alam M, Major S, Kohl-Bareis M, Petzold GC, et al. Endothelin-1-induced spreading depression in rats is associated with a microarea of selective neuronal necrosis. Exp Biol Med (Maywood). 2007; 232(2): 204-13.
مصرف اسيرى استنشاقى سوماترييتان مى تواند موجب كاهش مثري

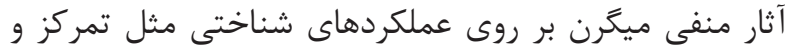

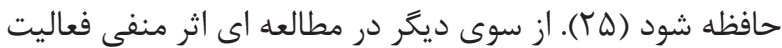

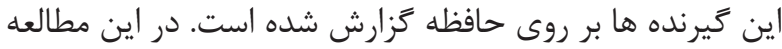

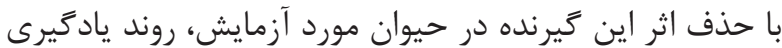

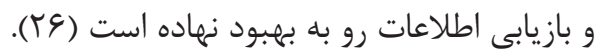

در مطالعهُ حاضر SD توانايى خود را در تخريب حافظةٌ اجتنابى

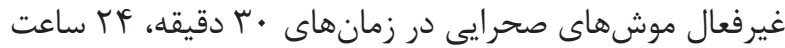

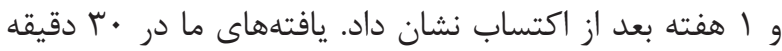

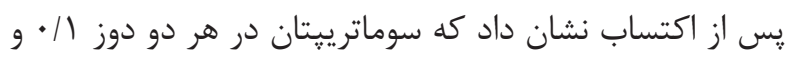

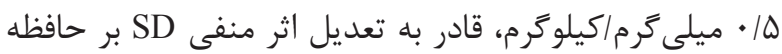

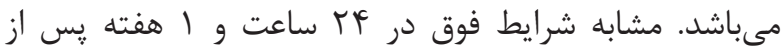

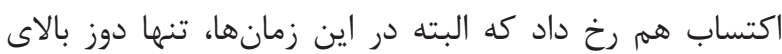

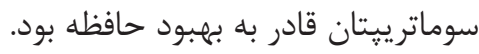

اين مسأله تأييدكننده آن است كه سوماترييتان تنها در شرايط

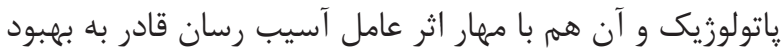

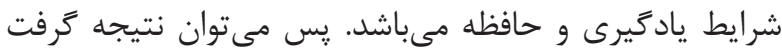

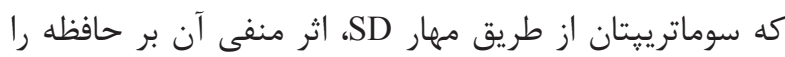
هم مهار كرده است.

مطالعات نشان دادهاند غلظتهاى پايين سروتونين مى مئواند

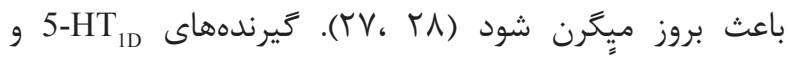
5-HT 1B

منابع

7. Yanamoto H, Miyamoto S, Tohnai N, Nagata I, Xue J-H, Nakano Y, et al. Induced spreading depression activates persistent neurogenesis in the subventricular zone, generating cells with markers for divided and early committed neurons in the caudate putamen and cortex. Stroke. 2005; 36(7): 1544-50.

8. Jafarian M, Rahimi S, Behnam F, Hosseini M, Haghir H, Sadeghzadeh B, et al. The effect of repetitive spreading depression on neuronal damage in juvenile rat brain. Neuroscience. 2010; 169(1): 388-94.

9. Lotfinia M, Lotfinia A, Khodaie B, Ahmadi M. Spreading depression enhances the rate of neurogenesis in the rat hippocampus and dentate gyrus. Eur J Neurol; 2014; $21: 264$.

10. Urbach A, Redecker C, Witte OW. Induction of neurogenesis in the adult dentate gyrus by cortical spreading depression. Stroke. 2008; 39(11): 3064-72.

11. Khodaie B, Lotfinia A, Ahmadi M, Lotfinia M, Jafarian M, Karimzadeh F, et al. Structural and functional effects of social isolation on the hippocampus of rats with traumatic brain injur. Behav Brain Res. 2014; 278C: 55-65. 
12. Morris R, Garrud P, Rawlins J, O'Keefe J. Place navigation impaired in rats with hippocampal lesions. Nature. 1982; 297(5868): 681-3.

13. Sutherland RJ, Kolb B, Whishaw IQ. Spatial mapping: definitive disruption by hippocampal or medial frontal cortical damage in the rat. Neurosci Lett. 1982; 31(3): 271-6.

14. Clark RE, Broadbent NJ, Squire LR. Hippocampus and remote spatial memory in rats. Hippocampus. 2005; 15(2): 260-72.

15. Sadeghian H, Jafarian M, Karimzadeh F, Kafami L, Kazemi H, Coulon P, et al. Neuronal death by repetitive cortical spreading depression in juvenile rat brain. Exp Neurol. 2012; 233(1): 438-46.

16. Lotfinia M. Memory impairement caused by spreading depression modulated by injection of nifedipine. Epilepsia. Eur J Neurol; 2014; 21: 589.

17. Ayata C, Jin H, Kudo C, Dalkara T, Moskowitz MA. Suppression of cortical spreading depression in migraine prophylaxis. Ann Neurol. 2006; 59(4): 652-61.

18. Costa-Cruz RRG, Amâncio-dos-Santos Â, Guedes RCA. Characterization of cortical spreading depression in adult well-nourished and malnourished rats submitted to the association of pilocarpine-induced epilepsy plus streptozotocin-induced hyperglycemia. Neurosci Lett. 2006; 401(3): 271-5.

19. Baluchnejadmojarad T, Roghani M. Chronic epigallocatechin-3-gallate ameliorates learning and memory deficits in diabetic rats via modulation of nitric oxide and oxidative stress. Behav Brain Res. 2011; 224(2): 305-10.

20. Jung SW, Han OK, Kim SJ. Increased expression of $\beta$ amyloid precursor gene in the hippocampus of streptozotocin-induced diabetic mice with memory deficit and anxiety induction. J Neural Transm. 2010; 117(12): 1411-8.

21. Khan MB, Hoda MN, Ishrat $\mathrm{T}$, Ahmad S, Khan MM, Ahmad A, et al. Neuroprotective efficacy of Nardostachys jatamansi and crocetin in conjunction with selenium in cognitive impairment. Neurol Sci. 2012; 33(5): 1011-20.

22. Egan MF, Kojima M, Callicott JH, Goldberg TE, Kolachana BS, Bertolino A, et al. The BDNF val66met polymorphism affects activity-dependent secretion of BDNF and human memory and hippocampal function. Cell. 2003; 112(2): 257-69.

23. King MV, Marsden CA, Fone KC. A role for the 5-HT(1A), 5-HT4 and 5-HT6 receptors in learning and memory. Trends Pharmacol Sci. 2008; 29(9): 482-92.

24. Meneses A. Physiological, pathophysiological and therapeutic roles of 5-HT systems in learning and memory. Rev Neurosci. 1998; 9(4): 275-89.

25. Farmer K, Cady R, Bleiberg J, Reeves D, Putnam G, O'Quinn S, et al. Sumatriptan nasal spray and cognitive function during migraine: results of an open-label study. Headache. 2001; 41(4): 377-84.

26. Malleret G, Hen R, Guillou J-L, Segu L, Buhot M-C. 5-HT1B receptor knock-out mice exhibit increased exploratory activity and enhanced spatial memory performance in the Morris water maze. J Neurosci. 1999; 19(14): 6157-68.

27. Stefulj J, Bordukalo-Niksic T, Hecimovic H, Demarin V, Jernej B. Epilepsy and serotonin (5HT): variations of 5HT-related genes in temporal lobe epilepsy. Neurosci Lett. 2010; 478(1): 29-31.

28. Deshmukh SV, Meyer JS. Cyclic changes in platelet dynamics and the pathogenesis and prophylaxis of migraine. Headache. 1977; 17(3): 101-8. 